\title{
Modelo para abordar la carga del cuidado de la enfermedad crónica en Colombia
}

\author{
A model for addressing the chronic disease care burden \\ in Colombia
}

\section{Modelo para tratar o encargo do cuidado da doença cronica na Colômbia}

\author{
Lucy Barrera-Ortiz ${ }^{1}$; Gloria M. Carrillo-González ${ }^{2}$; Lorena Chaparro-Díaz $^{3 *}$; \\ Beatriz Sánchez-Herrera ${ }^{4 *}$
}

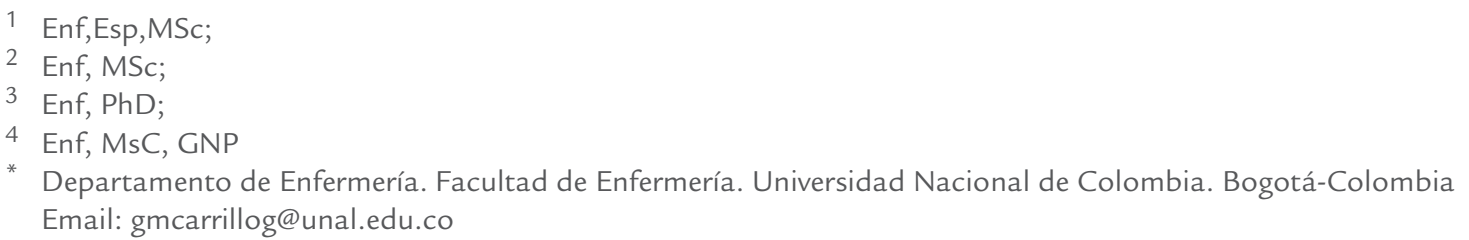

Recibido: abril 29 de 2013

Aceptado: abril 28 de 2015

\section{Resumen}

Con base en la experiencia, investigación y revisión de literatura, se construyo una propuesta de modelo para abordar la carga del cuidado de la enfermedad crónica que fue validado mediante prueba de expertos bajo criterios de comprensibilidad, claridad, coherencia, suficiencia, relevancia y pertinencia y sometido a prueba en campo con 144 profesionales de diferentes áreas de la salud en las cinco macro regiones de Colombia. El planteamiento de un modelo para abordar la carga del cuidado de la Enfermedad Crónica (EC) en Colombia y la propuesta de indicadores para operacionalizarlo (definiendo variables con factores medibles) con iniciativas relevantes con implementación de iniciativas sobre políticas en salud las cuales aseguran beneficios sa los pacientes y al sistema de salud en Colombia..

Palabras clave: Cuidadores, Familia, Costos de la enfermedad, Enfermedad Crónica, Colombia. Decs

\begin{abstract}
A proposal regarding a model for approaching the chronic disease (CD) care burden was constructed; it was based on experience, research and a review of the pertinent literature. Such model was validated by peer review (i.e. experts in the field) using criteria related to understandability, clarity, coherence, sufficiency, relevance and pertinence and then submitted to a field trial involving 144 professionals from different areas of the healthcare field drawn from five macro-regions in Colombia. Such approach regarding a model for addressing the burden of caring for people suffering from CD in Colombia and the proposed indicators for operationalising it (i.e. strictly defining variables into measurable factors) should lead to relevant health policy initiatives being implemented which should ensure health benefits for patients and the Colombian health system.
\end{abstract}

Key words: Caregiver, family, cost of chronic disease, chronic disease, Colombia (Decs). 


\begin{abstract}
Resumo
Com base na experiência, pesquisa e revisão foi construda uma proposta de modelo para discutir o trabalho do cuidado da doenca cronica, que foi validado mediante prova de expertos, sob criterios de comprensao, calidade, relevância e pertinencia, testado em campo com 144 profissionais de diferentes áreas de saúde nas cinco macro regiões da Colômbia. A abordagem de um modelo para discutir a atividade de cuidar da DC na Colômbia e a proposta de indicadores para operacionalizar (estabelecendo variáveis com fatores medíveis) com iniciativas relevantes e implementação de politicas em saúde as quais proporcionam benefícios aos pacientes e ao sistema de saúde na Colômbia.
\end{abstract}

Palavras chave: Cuidadores, família, Doença Crônica, Efeitos Psicossociais da Doença, Colombia.

\section{Introducción}

El inesperado incremento de las enfermedades crónicas en América Latina (OMS, 2012), del cual Colombia no es una excepción (OMS, 2014), plantea la necesidad de actualizar la conceptualización y manejo del cuidado de la salud y exige para ello la revisión y ajuste de los modelos de atención en salud (Rogers, 2009) o el replanteamiento de los mismos.

Se encuentran reportados en la literatura diferentes modelos teóricos y conceptuales para el trabajo en el campo de la Enfermedad Crónica (EC) (Jova, et al., 2011) como: 1. Aquellos que explican los ajustes a una enfermedad discapacitante como la artritis reumatoidea (Walker, Jackson y Littlejohn, 2004) los que explican la conducta de los fumadores, cuando tienen o no enfermedad crónica (Wagner, Heapy, Frantsve, Abbott y Burg, 2006), los que explican la experiencia de la enfermedad terminal en diferentes escenarios (Byock, Norris, Curtis y Patrick, 2001) y (Nolan y Mock, 2004), el modelo de autorregulación del dolor crónico (Hobro, Weinman y Hankins, 2004), el modelo para el manejo de la diabetes con apoyo telefónico (Lange, et al., 2010), el modelo de cuidado paliativo aplicado a personas con enfermedad de Parkinson (Roger, 2012) y el modelo que va de dependencia e independencia en las situaciones de EC (Gignac y Cott 1998). 2. Los modelos de trabajo con cuidadores familiares de personas con EC que incluyen aquellos que consideran sus necesidades de información (Washington, Meadows, Elliott y Koopman, 2011), sus necesidades de soporte (Cameron y Gignac, 2008), y el efecto de los cuidadores en los resultados de la salud (Cho, 2007). 3. Los modelos que se basan en el cuidado como el de toma de decisiones para seguimiento telefónico (Hebert, Korabek y Scott, 2006), la comprensión de las transiciones dentro de la EC (Blum y Sherman, 2010), el modelo para mejorar la evaluación del cuidado de la EC (Baker, et al, 2005), el modelo de abordaje por tareas (Samson y Siam, 2008) y el de comprensión de la enfermedad crónica misma (Kelvyn y Craig 1995). Sin embargo, no aparece reportado en la literatura ningún modelo para abordar la carga del cuidado de la EC.
Con el fin de dar una respuesta adecuada a las demandas de las personas con EC, sus cuidadores familiares, los profesionales e instituciones de salud y al Sistema General de Seguridad Social en Salud (SGSSS) mismo, este trabajo planteó y validó un modelo para abordar dicha carga aplicándolo a Colombia, teniendo como propósito final su aplicación para disminuir la carga del cuidado.

El desarrollo del modelo para abordar la carga del cuidado de la EC en Colombia buscó un enfoque integral por ser un modelo basado en el cuidado (Barrera, Pinto y Sánchez, 2008).

Puede señalarse que según la clasificación de modelos propuesta por Bausela (2004), el presente es un modelo: 1. funcional, es decir sencillo, práctico y utilizable. 2. De orientación humanista. 3. Con una intervención propuesta en términos de servicio. 4. De sucesión, pues presenta etapas para llegar a una finalidad. 5. Representativo, puesto que reproduce las características de la carga del cuidado. 6 . Predictivo, ya que anuncia los posibles resultados de un cambio que está por venir, partiendo del grado de éxito de individuos en un contexto y proceso determinados. 7. Unitario, acepta la condición inseparable de quienes participan en esta experiencia de cuidado de la persona con EC para trascender de la comprensión de las formas a la comprensión de los hechos.

\section{Metodología}

Estudio descriptivo de diseño y validación de un modelo, desarrollado como parte del Programa para la Disminución de la Carga de la EC en Colombia entre los años 2012 y 2014 en 10 pasos secuenciales tal como se ilustra en la Figura 1.

En 1996 y como respuesta al cambio del perfil epidemiológico regional, la Universidad Nacional de Colombia conformó el Grupo de Cuidado al Paciente Crónico y su Familia para fortalecer la docencia, la investigación y la extensión universitaria en el campo. A partir de ello se han construido nueve asignaturas del 


\section{METODOLOGÍA PARA LA CONSTRUCCIÓNY VALIDACIÓN DEL MODELO}

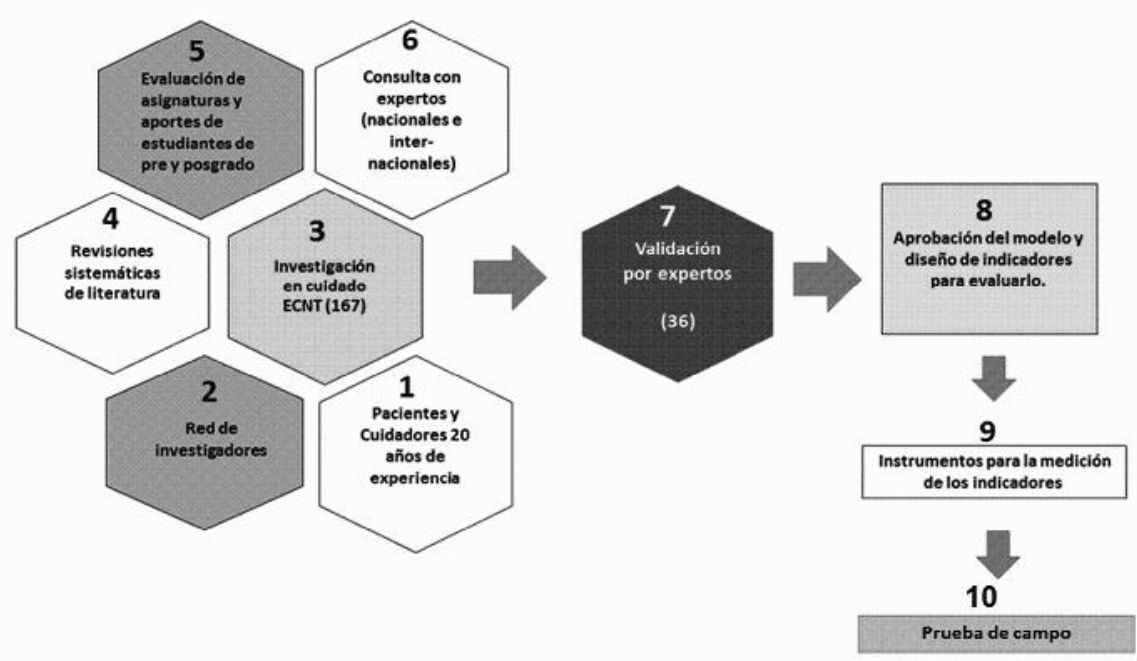

Fuente: Elaboración propia.

Figura 1. Pasos para la construcción y validación del modelo para abordar la carga de la enfermedad Crónica

área, para programas de pre y posgrado que desde la Facultad de Enfermería se ofrecen a las diferentes disciplinas de la Universidad.

Durante este periodo y gracias a la conformación de la Red Latinoamericana de Cuidado al paciente crónico (RED), se diseñó y se puso en marcha el Programa Cuidando a los Cuidadores@ (Barrera, Pinto y Sánchez, 2006) que ha generado contacto asistencial e investigativo con personas que viven la experiencia del cuidado en situaciones crónicas de enfermedad desde diferentes ángulos y en diferentes escenarios.

En el campo de la investigación la RED ha realizado 167 estudios sobre diferentes aspectos del cuidado de las personas con EC en el ámbito nacional e internacional. Con base en ellos, se cuenta con una caracterización de las personas con EC, los cuidadores familiares, los profesionales y las instituciones que en conjunto apoyan la evidencia de la preparación insuficiente de los SGSSS de América Latina frente a la creciente demanda de cuidado de las personas con EC (OMS, 2012).

Dentro de los avances logrados ha sido posible evidenciar que el cuidado de las personas con EC genera carga que si bien ha sido descrita desde varios ángulos, aún no es bien comprendida. La literatura reporta medidas para analizar la carga de la EC en diferentes países del mundo incluidos varios de América Latina (Ministerio de salud de Chile y Universidad Católica de Chile, 2007), (Ministerio de salud del Perú, 2008), (Rodríguez, Acosta y Peñaloza, 2008), (Lozano, Franco y
Solís, 2008), (Borruel, Mas y Borruel, 2010) y (Lozada, Aguinaga, Páez, Olmedo y Pozo, 1995). Los diversos estudios consideran la carga de la EC como un fenómeno desintegrado de la vida cotidiana de estas personas y de quienes con ellas comparten la experiencia de vivir con su EC. La comprensión de la carga del cuidado de la EC supera en gran medida la información epidemiológica disponible ydebe responder a un paradigma integral que permita abrir caminos en la búsqueda de alternativas para cualificar los SGSSS de la región.

La evidencia disponible indica que las personas con EC la mayor parte del tiempo son atendidas por sus familiares (Barrera, Pinto y Sánchez, 2006) con repercusiones en la funcionalidad y la salud de estas familias (Vinaccia y Orozco, 2005), (Gonzales, et al., 2002), Achury, Castaño, Gómez y Guevara, 2012) y (Ariza, et al., 2010) y que ellas tienen dificultades con los profesionales de la salud quienes en muchos casos no se encuentran preparados para este tipo de atención (Barrera, Pinto y Sánchez, 2006) y (Córdova, et al., 2008), y con el SGSSS que a pesar de algunos esfuerzos locales (Lucumí, et al., 2008), no ha puesto en marcha una respuesta concreta para el cuidado a largo plazo que requieren las personas con EC mostrando así su vulnerabilidad como sistema (Alwan y Etienne, 2010).

Medir la carga del cuidado de las personas con EC permitirá entre otras, dar respuestas a las múltiples necesidades de quienes viven la experiencia de la EC y buscar disminuir dicha carga. 
Se incluyó posteriormente una revisión de la literatura con respecto a modelos de cuidado en situaciones de enfermedad crónica, seleccionado los más pertinentes para este propósito o aquellos que hubiesen sido validados. La revisión se hizo bajo los descriptores Cuidado, Enfermedad o padecimiento crónico y modelos, con sus respectivas traducciones. La ventana de observación de fue de 20 años, en idiomas inglés y español y con el empleo de la herramienta Descubridor del Sistema de Bibliotecas de la Universidad Nacional de Colombia que integra 14 bases de datos reconocidas por su prestigio académico.

A la información anterior se le sumó un análisis transversal de la evaluación de las asignaturas de pregrado y posgrado sobre el tema. Este permitió acoger algunas sugerencias que apoyaron el diseño preliminar del modelo.

Se procedió luego a una consulta con expertos latinoamericanos en cuidado a personas con EC y con base en ella, se plasmó el modelo propuesto.

El siguiente paso fue la validación que se desarrolló con la participación de 36 expertos de diferentes áreas de la salud en dos sesiones consecutivas en las que se revisó si el modelo era comprensible, claro, coherente, suficiente, relevante y esencial para poder abordar la carga del cuidado con el fin de disminuirla. Esta validación incluyó la revisión de los indicadores y propuestas de instrumentos de medición por parte de los autores. Ver tabla 1.

Finalmente, se complementó la anterior validación con una prueba de campo en la cual participaron 144 profesionales de diferentes áreas de la salud en las cinco macro regiones de Colombia. Esta prueba se orientó y acompañó con las guías establecidas para un diplo- mado que incluyó los roles administrativo - directivo y asistencial y previó competencias de tipo cognitivo, instrumental y sistémico para garantizar su comprensión, aplicación y medición del mejoramiento de la práctica.

Aspectos éticos. Este proyecto mantiene todos los requisitos exigidos en la Resolución No.8430 de 1993, por la cual se establecen las normas científicas, técnicas y administrativas para la investigación en salud en Colombia (Ministerio De Salud de la República de Colombia, 1993).

Aspectos ambientales. En el compromiso con el medio ambiente se acogió la política ambiental de la Universidad Nacional de Colombia, estipulada en el Acuerdo 16 de 2011 del Consejo Superior Universitario, en todos los aspectos pertinentes y en particular en el uso responsable de recursos y el ejemplo y exigencia que sobre ello se tiene con los estudiantes y auxiliares de investigación (Universidad Nacional de Colombia, 2011).

\section{Resultados}

El modelo. A continuación se presentan el modelo propuesto, sus conceptos, supuestos, estructura interna, contexto y dinámica para finalizar con la propuesta de indicadores de evaluación.

Conceptos del Modelo. Son conceptos esenciales dentro del modelo: 1) El cuidado, 2) la experiencia de la EC, 3) la carga y, 4) la calidad de vida. Son conceptos complementarios 5) la habilidad de cuidado, 6) el soporte social y 7) las Tecnologías de información y comunicación (TIC). Ver figura 2.

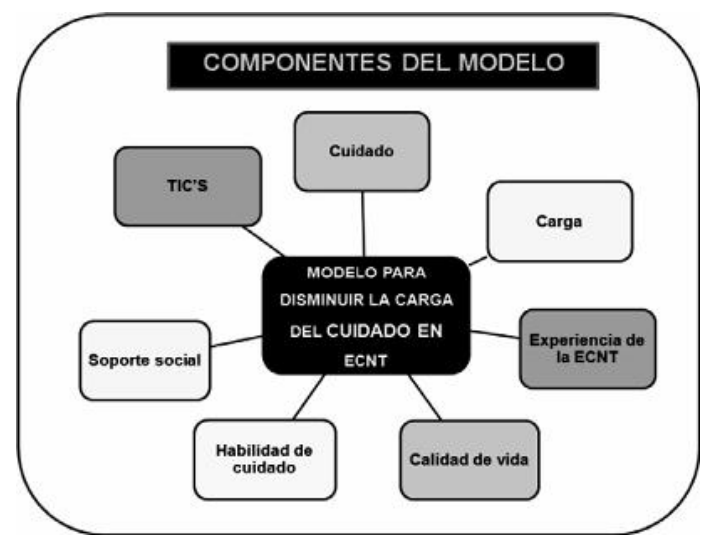

Fuente: Elaboración propia.

Figura 2. Conceptos esenciales del modelo para abordar la carga del cuidado de la EC en Colombia 
Tabla 1. Características de los expertos que participaron en el proceso de validación

\begin{tabular}{|c|c|c|c|c|}
\hline País & Ciudad & $\begin{array}{c}\text { No. de } \\
\text { expertos }\end{array}$ & Área de conocimiento & Principales aportes \\
\hline \multirow{6}{*}{ Colombia } & Bogotá & 16 & $\begin{array}{l}\text { Cuidado a la persona } \\
\text { con EC (enfermería, } \\
\text { medicina, } \\
\text { farmacia, economía, } \\
\text { trabajo social, } \\
\text { epidemiología, } \\
\text { psicología, derecho) }\end{array}$ & $\begin{array}{l}\text { Contextualizar el modelo frente a las características del } \\
\text { sistema general de salud en Colombia (barreras, riesgos, entre } \\
\text { otros) } \\
\text { Clarificar los conceptos de carga que son aplicables al } \\
\text { programa ya que se tienen diferentes abordajes desde las } \\
\text { disciplinas. } \\
\text { Clarificar el concepto de calidad de vida que es aplicable al } \\
\text { modelo ya que se tiene variedad de abordajes. } \\
\text { Incluir aspectos de evaluación de percepciones culturales. } \\
\text { Incluir el papel institucional en el cuidado en casa (relación } \\
\text { institución-cuidador) } \\
\text { Revisar la nueva conceptualización de salud de la OMS vs } \\
\text { Global Burden of Disease. } \\
\text { Contemplar asociaciones de paciente/cuidador/profesional } \\
\text { en el uso de TIC VS efectividad. } \\
\text { Hacer más explícito el concepto de integralidad }\end{array}$ \\
\hline & Pasto & 5 & $\begin{array}{l}\text { Cuidado a la persona } \\
\text { con EC }\end{array}$ & $\begin{array}{l}\text { Clarificar algunos conceptos como institución atractiva y } \\
\text { hospital magnético. } \\
\text { Abordar el cuidado interdisciplinario en la población objeto } \\
\text { de estudio. } \\
\text { Tener en cuenta a las personas con EC en situación de } \\
\text { discapacidad } \\
\text { Contemplar los programas a nivel institucional para } \\
\text { potenciarlos con el modelo. } \\
\text { Contemplar el flujo de información de bases de datos de } \\
\text { pacientes. }\end{array}$ \\
\hline & Cartago & 1 & $\begin{array}{l}\text { Cuidado a la persona } \\
\text { con EC }\end{array}$ & $\begin{array}{l}\text { Incluir en los supuestos básicos del modelo que la percepción } \\
\text { de carga en el cuidador familiar puede disminuir si las } \\
\text { responsabilidades de cuidado son compartidas. }\end{array}$ \\
\hline & Sangil & 2 & $\begin{array}{l}\text { Cuidado a la persona } \\
\text { con EC }\end{array}$ & $\begin{array}{l}\text { Cambiar la denominación paciente por persona con EC } \\
\text { Incluir la capacitación a enfermeras } \\
\text { Mirar de forma específica las intervenciones de enfermería }\end{array}$ \\
\hline & Cundinamarca & 5 & $\begin{array}{l}\text { Cuidado a la persona } \\
\text { con EC }\end{array}$ & Contemplar el SGSSS \\
\hline & Bucaramanga & 2 & $\begin{array}{l}\text { Cuidado a la persona } \\
\text { con EC }\end{array}$ & $\begin{array}{l}\text { Sugieren que en el apartado de antecedentes y justificación } \\
\text { se deje ver los vacíos existentes que hay en los modelos con } \\
\text { los que se ha abordado históricamente la carga. } \\
\text { Sugieren que todos los componentes del modelo de carga se } \\
\text { ubiquen alrededor del cuidado para disminuir la carga } \\
\text { Revisar si el componente del uso de TICs es el que potencia } \\
\text { todos los demás componentes del modelo o todos } \\
\text { interactúan a un mismo nivel. } \\
\text { Ampliar el abordaje metodológico del modelo } \\
\text { Considerar la posibilidad de mirar el abordaje familiar } \\
\text { Evidenciar las intervenciones de enfermería cuando se hable } \\
\text { de abordaje profesional }\end{array}$ \\
\hline Guatemala & $\begin{array}{l}\text { Ciudad de } \\
\text { Guatemala }\end{array}$ & 2 & $\begin{array}{l}\text { Cuidado a la persona } \\
\text { con EC }\end{array}$ & Contemplar la reciprocidad en la definición de cuidado. \\
\hline México & $\begin{array}{l}\text { Ciudad de } \\
\text { México }\end{array}$ & 2 & $\begin{array}{l}\text { Cuidado a la persona } \\
\text { con EC }\end{array}$ & $\begin{array}{l}\text { Buscar instrumentos validados y aplicables al personal de } \\
\text { enfermería, a los pacientes y empleadores. } \\
\text { Hacer listas de cotejo logístico de las instituciones. } \\
\text { Realizar capacitación a grupo líder de enfermería en el } \\
\text { modelo. } \\
\text { Validar el modelo mediante unidades piloto. } \\
\text { Establecer cronograma de evaluación de resultados. }\end{array}$ \\
\hline EEUU & Boston & 1 & $\begin{array}{l}\text { Cuidado a la persona } \\
\text { con EC. Odontólogo }\end{array}$ & $\begin{array}{l}\text { Traducir el modelo a inglés } \\
\text { Presentarlo a otros profesionales de salud para complementar } \\
\text { la visión. }\end{array}$ \\
\hline
\end{tabular}

Fuente: Datos de la investigación. 
(1) El cuidado. El cuidado implica la interacción de unos sujetos en un contexto con el fin último de lograr el desarrollo como personas y crecer en su capacidad como cuidadores (Boykin y Schoenhofer, 2000). Este cuidado además de buscar mejorar el bienestar y ser bien intencionado, debe ser seguro, es decir, confiable subjetiva y objetivamente, teóricamente avanzado, técnicamente sólido, demostrable y susceptible de mejoramiento, (Sánchez, 2010). Los problemas del cuidado de las personas con EC se dividen en cuatro grandes grupos que incluyen: la necesidad de modificar el estilo de vida para mejorar el pronóstico: el manejo del dolor, la limitación y la mutilación; afrontar las alteraciones en la capacidad de socializar; y el afrontamiento con la muerte o una gran pérdida. (Barrera, et al., 2010)

(2) La experiencia de la EC. Para una persona vivir en situación de enfermedad crónica es identificarse a sí mismo como alguien que se mueve en expresiones de enfermedad con permanencia en el tiempo, que le generan incapacidad residual, es admitir la existencia de un trastorno que tiende a generar discapacidad permanente y que exige la modificación del estilo de vida. Se asocia con una alteración irreversible y que hace que requiera cuidados especiales o paliativos por cuanto no se conoce curación para la misma. Las personas que viven en estas situaciones son seres activos y trascendentes, que tienen la capacidad de crecer en el cuidado. Esta vivencia se percibe por el individuo como una disfunción para cumplir con su rol, según parámetros esperados y que le ha acompañado por más de seis meses. Para el cuidador familiar la experiencia de la EC significa un cambio en su rol, una responsabilidad adicional, una experiencia de cuidado como parte de una familia.Para la Diada Cuidador - Persona con EC, un vínculo nuevo, un plan de vida compartido, una mirada conjunta al cuidado. Para el profesional de la salud un reto de aprendizaje, un paciente que no mejora y requiere cuidado. Para las instituciones la necesidad de un ajuste a las prácticas de atención actuales, una dinámica de seguimiento, un reto para el servicio. Para el SGSSS, la necesidad de repensarse para atender la realidad de sus usuarios que viven la experiencia del cuidado de una EC. (Barrera, et al., 2010)

(3) La carga. Carga significa trabajo, dificultad, peso, desgaste, energía necesaria para algo. Cuando se habla de la carga del cuidado de la EC se hace referencia al esfuerzo adicional que implica para la persona con la EC, sus cuidadores familiares o profesionales satisfacer las necesidades de cuidado. Esta carga no puede ser totalmente medida o cuantificada al igual que los factores contextuales que la aumentan o disminuyen, sin embargo es fundamental comprender su dinámica para abordar de manera adecuada el cuidado de la salud en las situaciones de EC. (Sánchez, et al., 2013)
(4) La calidad de vida. Calidad de vida es el conjunto de condiciones subjetivas y objetivas que caracterizan la existencia de una persona, que pueden ser positivos o negativos y han sido agrupados en cuatro dimensiones según su nivel de bienestar: la física, que incluye la fisiología, la funcionalidad y la sintomatología que presenta el sujeto. La psicológica que es la suma del afecto, la sensación de control, de temor y de felicidad. La social que se compone de las interrelaciones, la sociabilidad, la vida familiar, la expresión de la sexualidad y los recursos de que se dispone. La espiritual que encierra el significado y propósito de la vida, el sentido de esperanza y el de trascendencia (Ferrell, et al., 1995) y (Carreño, 2012).

(5) Habilidad de cuidado. Habilidad de cuidado es el potencial de cuidado que tiene la persona que se encuentra en situación de enfermedad crónica, su familiar o el profesional responsable de su atención. Esta habilidad incluye la dimensión cognoscitiva y la actitudinal que pueden ser identificadas y medidas mediante el conocimiento o dominio del tema, valor o forma de relacionarse, apoyarse y tomar decisiones y paciencia o significado que se dé a la situación (Nkongho, 1990); (Carrillo, Sánchez y Barrera, 2014).Vista así, la habilidad puede medirse a través de la comunicación directa con cada persona.

(6) Soporte social. El soporte social es el recurso externo con que se cuenta para abordar las necesidades de cuidado. Este cumple un papel determinante como efecto mediador dentro de las experiencias vitales estresantes y la enfermedad y puede reducir significativamente el impacto percibido por esta última. El soporte social favorece una mayor adaptación a las situaciones, con repercusiones directas en la calidad de vida de las personas que padecen las dolencias crónicas (Carrillo, Chaparro, Barrera, Pinto y Sánchez, 2011). En este soporte se reconocen cuatro componentes fundamentales: 1) la red social, 2) la interacción social, 3) el soporte social percibido y 4) el soporte instrumental (Struk, Moss y Focus, 2009).

(7) Tecnologías de la información y la comunicación (TIC). Las TIC son todos aquellos elementos, herramientas y técnicas que se emplean para comunicar o informar algo. Ellas han demostrado tener utilidad en la difusión del conocimiento y en la sistematización de procesos, en particular del conocimiento y servicios de salud (Cárdenas, et al., 2010)

Los supuestos básicos del modelo. Los conceptos planteados interactúan dentro de este modelo de una manera particular. A continuación se presentan los 12 supuestos básicos que dejan ver su interacción: 
1. El cuidado se da entre personas concretas y en un contexto determinado e implica integralidad y unicidad.

2. La salud en la EC se asocia con la calidad de vida de la persona enferma y de su cuidador familiar.

3. El contexto y las transiciones inciden de manera importante en la calidad de vida de la persona con EC y de sus cuidadores.

4. El SGSSS es el contexto más amplio de la atención en salud para las personas con EC y este debe garantizar seguridad en el cuidado.

5. La experiencia de EC implica al SGSSS a través de la persona con la enfermedad, sus cuidadores familiares, los profesionales de la salud y las instituciones de salud.

6. La percepción de carga se asocia de manera importante con la calidad de vida de quienes viven la experiencia de la EC y la satisfacción de quienes suministran el servicio.

7. El soporte social puede facilitar el cuidado de las personas con EC, mejorar la calidad de vida y disminuir la percepción de carga del cuidado.

8. Las TIC pueden ser un medio útil para prestar soporte social a quienes viven la experiencia del cuidado de la EC.

9. Cuidar familiar o profesionalmente a las personas con EC implica habilidad.

10. La habilidad de cuidado permite fortalecer y aprovechar las opciones de soporte social.

11. Las instituciones se vuelven más atractivas cuando logran disminuir la carga del cuidado de la EC fortaleciendo la habilidad de cuidado de las personas con la enfermedad y la de sus cuidadores familiares y capacitando a sus profesionales.

12. El SGSSS debe responder a las necesidades del cuidado de las personas con EC y de sus cuidadores familiares.

La estructura interna del modelo. El modelo para abordar la carga del cuidado de la EC en Colombia refleja que los sujetos del cuidado que incluyen a las personas con EC, los cuidadores familiares, la diada cuidador receptor como sujeto y los profesionales de la salud pueden mejorar su nivel de soporte social o su nivel de información y capacitación si las instituciones apoyan el cuidado. Para este apoyo se consideran útiles las TIC disponibles. Este soporte e información o capacitación fortalecidos generan a su vez mayor habilidad de cuidado y mejor calidad de vida. Se genera un círculo virtuoso entre la mayor habilidad y el soporte social, de manera que cuanto más hábil el sujeto de cuidado, mayor aprovechamiento podrá tener del soporte disponible. Al tener mayor habilidad se disminuyen los riesgos innecesarios, los costos del cuidado y se incrementa su nivel de seguridad con la consecuencia total de tener una menor carga del cuidado. En la medida en que las instituciones logren fomentar este tipo de apoyo a las personas que viven la experiencia del cuidado de la EC se volverán más atractivas generando magnetismo frente a posibles usuarios. Ver figura 2

El contexto del modelo. Para las personas con EC y sus familias el SGSSS actualmente es adverso. Desde la perspectiva del usuario con una EC la atención es fraccionada, desintegrada, dificulta los procesos exigiendo múltiples autorizaciones para diferentes problemas relacionados con la EC, no considera ni apoya a los cuidadores familiares, no se encuentra preparado para abordar la EC, incrementa las transiciones innecesarias y con ello el costo, el riesgo y la inseguridad de las personas con EC y no tiene propuestas para ajustarse a la realidad que vive el país frente al incremento de estas enfermedades. Frente a este contexto para disminuir la carga del cuidado de la EC se espera que cada uno de los actores del cuidado tenga unos logros individuales y la suma de ellos genere un impacto positivo sobre el SGSSS.

Serán indicadores de logro en este proceso: 1) Para las personas con EC que estén mejor informados, más apoyados, tengan mayor nivel de seguridad en su cuidado y en consecuencia una mejor calidad de vida. 2) Con los Cuidadores familiares de las personas con EC se espera que sean más hábiles en el cuidado, tengan mejor soporte social, menores costos del cuidado y al igual que las personas con EC una mejor calidad de vida. 3) De los profesionales se espera un mejor nivel de preparación frente al reto que asumen, un mayor nivel de información, mayor satisfacción con lo que hacen en su trabajo y que estén brindando un cuidado más seguro. 4) En el caso de las instituciones se espera que hagan un mejor uso de las $\mathrm{TIC}$, den un cuidado con calidad y mayor seguridad y sin duda que se conviertan en unas instituciones más atractivas para sus usuarios. Se espera que al aplicar el modelo para abordar la carga esta se reconozca, se pueda disminuir o atenuar y de esta forma genere cualificación en el sistema. Ver figura 3

Indicadores de medición del modelo. El modelo considera indicadores de logro frente a cada uno de los 


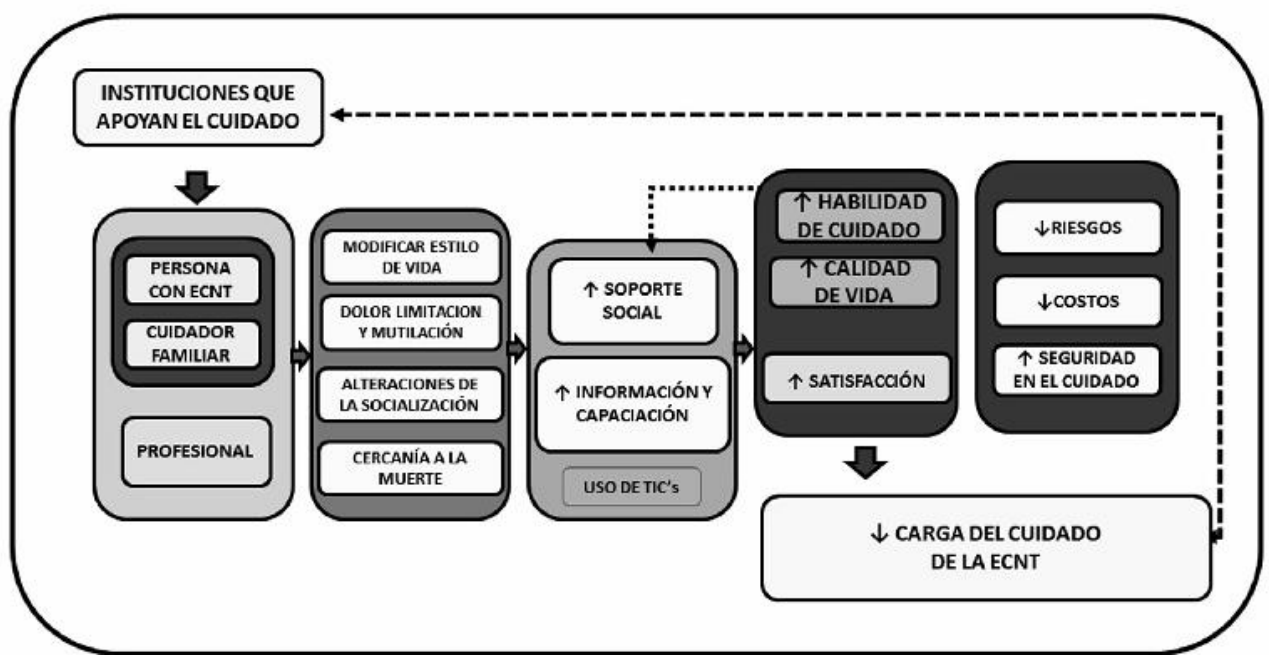

Fuente: Elaboración propia.

Figura 2. Estructura interna del modelo para abordar la carga del cuidado de personas con EC

integrantes de la experiencia del cuidado de la EC incluyendo a la persona con la enfermedad, a su cuidador familiar y a los dos como diada, los profesionales de la salud, las instituciones y el SGSSS. Ver figura 4.

\section{Validación}

A juicio de los expertos el modelo requirió unos ajustes en su representación gráfica y una vez realizados fe evaluado como comprensible, claro, coherente, suficiente, relevante y esencial para poder abordar la carga del cuidado con el fin de disminuirla.

Los resultados esperados se transformaron en indicadores bajo la metodología UN SIMEGE y cada uno de ellos dejó previstos los instrumentos de medición requeridos para poderlos reportar.

Finalmente, para aplicar el modelo en campo se desarrolló un diplomado ofertado a 144 profesionales de diferentes áreas de la salud en las cinco macro regiones de Colombia que incluyó los componentes del modelo para el cual cada uno de los participantes debía cumplir competencias de tipo cognitivo, instrumental y sistémico. La evaluación de dicho diplomado señaló que el modelo fue comprendido, pudo ser aplicado en todas las regiones y permitió además de medir, cualificar la práctica.

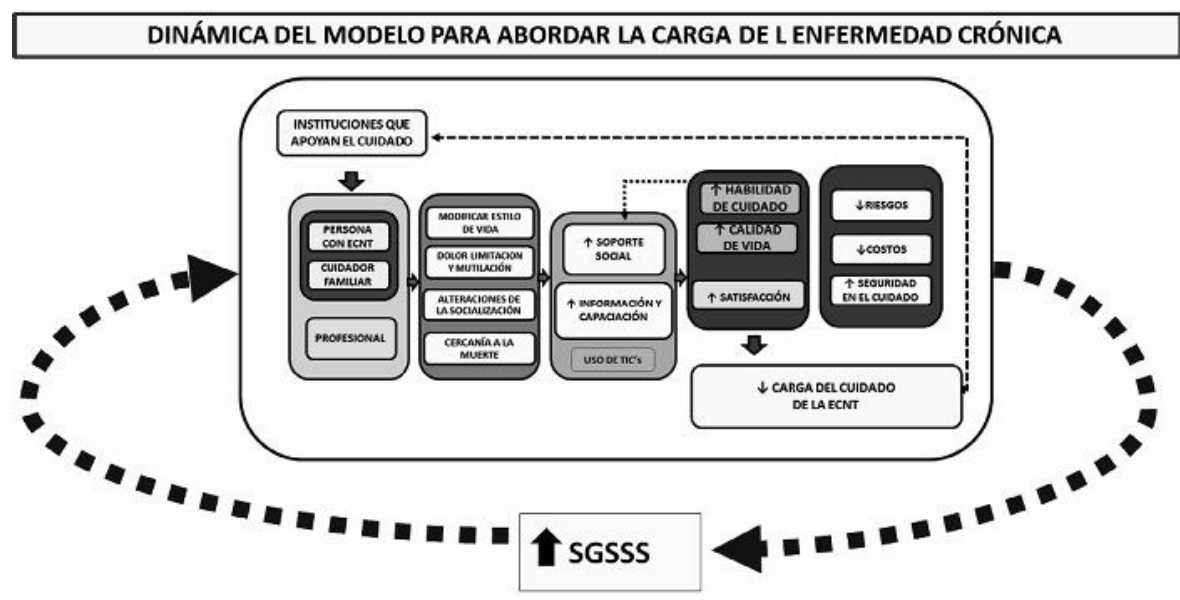

Fuente: elaboración propia

Figura 3. Dinámica del modelo de abordaje de la carga del cuidado de personas con EC 


\section{LA CONSTRUCCIÓN DE LOS INDICADORES DEL MODELO: RESULTADOS ESPERADOS}

\begin{tabular}{|c|c|c|c|c|}
\hline PACIENTE & CUIDADOR & PROFESIONAL & INSTITUCIÓN & SISTEMA \\
\hline $\begin{array}{l}\text { Mejor } \\
\text { informado }\end{array}$ & Más hábil & $\begin{array}{l}\text { Más y mejor } \\
\text { preparado }\end{array}$ & $\begin{array}{l}\text { Mejor uso de } \\
\text { TIC's }\end{array}$ & $\begin{array}{c}\text { Mayor claridad en } \\
\text { situación de los usuarios }\end{array}$ \\
\hline Más apoyado & $\begin{array}{l}\text { Mejor soporte } \\
\text { social }\end{array}$ & Más satisfecho & $\begin{array}{l}\text { Mayor nivel } \\
\text { de calidad }\end{array}$ & $\begin{array}{l}\text { Fortalecimiento de } \\
\text { comunidades de cuidado }\end{array}$ \\
\hline $\begin{array}{l}\text { Mejor calidad de } \\
\text { vida }\end{array}$ & $\begin{array}{c}\text { Mejor calidad de } \\
\text { vida }\end{array}$ & Más seguro & $\begin{array}{l}\text { Mayor nivel de } \\
\text { seguridad }\end{array}$ & $\begin{array}{l}\text { Revisión de las políticas } \\
\text { de cuidado crónico y } \\
\text { paliativo }\end{array}$ \\
\hline Más seguro & Menor costo & Más competente & Más atractiva & $\begin{array}{l}\text { Calidad en el cuidado de } \\
\text { la salud en situaciones de } \\
\text { Enfermedad Crónica }\end{array}$ \\
\hline \multicolumn{5}{|c|}{ MENOR CARGA } \\
\hline
\end{tabular}

Fuente: Elaboración propia

Figura 4. Indicadores del modelo de abordaje de la carga del cuidado de personas con EC

\section{Discusión}

Abordar la carga del cuidado de la enfermedad crónica (EC) es un fenómeno complejo, pero sin duda necesario para buscar disminuir dicha carga. Este trabajo implica abrir un camino nuevo que complemente las actuales mediciones de la carga de la EC bajo un paradigma integral de cuidado. La diferencia entre las mediciones existentes y las necesarias, es que las primeras generan según lo reflejan los diferentes estudios de América Latina datos epidemiológicos, como los años de vida productivos perdidos (AVISAS), años perdidos por discapacidad (APD) y años perdidos por muerte prematura (AVPM), entre otros (Ministerio de salud de Chile y Universidad Católica de Chile, 2007), (Ministerio de salud del Perú, 2008), (Rodríguez, Acosta y Peñaloza, 2008), (Lozano, Franco y Solís, 2008), (Borruel, Mas y Borruel, 2010) y (Lozada, Aguinaga, Páez, Olmedo y Pozo, 1995). Las segundas, retoman estos parámetros y los complementan con la forma como estos años de vida afectan la calidad de vida de la persona enferma, las de su cuidador familiar y el impacto de esta situación en el contexto sistema general de seguridad social en salud (SGSSS), es decir, consideran la experiencia del cuidado de la salud humana integralmente. Varios autores señalan que bajo el paradigma del cuidado integral, esta experiencia del cuidado de la salud puede ser comprendida (Grupo de
Cuidado, 1998). El cuidado aportará un avance para que las personas encuentren su lugar en el mundo en lugar de estar en busca permanente de un lugar (Mayeroff, 1190), para que la vida más consciente sea una expresión de la salud (Newman, 2008) y para que se logre que la calidad de vida supere el concepto cuantificable para volverse una realidad (Ferrell, et al., 1995), (Carreño, 2012).

Por otro lado, se espera revisar además de los indicadores epidemiológicos convencionales otros aspectos que reflejen la realidad de la vida cotidiana de la persona enferma y su cuidador familiar, la preparación y disponibilidad de los profesionales de salud, de las instituciones y del contexto mismo en donde la academia tiene un rol fundamental en generar propuestas que atiendan las necesidades de los usuarios de los SGSSS. Este modelo prevé una retroalimentación permanente para que el SGSSS colombiano se cualifique en respuesta a las cambiantes necesidades de una población que día a día tiene mayor número de personas con enfermedades crónicas (OMS, 2012), y responde a las prioridades identificadas por expertos en el campo de la salud en Colombia (Agudelo, et al., 2009).

La validación de los expertos y la prueba de campo permiten señalar que el modelo tiene una alta significancia social y teórica frente al cuidado de la experien- 
cia de la EC que es una respuesta contextualizada que atiende el llamado de la OMS para buscar estrategias de disminuir la carga de la EC en América Latina.

\section{Conclusión}

El modelo presentado para abordar la carga del cuidado de la EC en Colombia y la propuesta de indicadores para operacionalizarlo son una herramienta útil para avanzar en el camino del estudio de la EC y sus implicaciones para las personas con EC, sus cuidadores familiares, los profesionales, las instituciones y el mismo SGSSS. El proceso de validación internacional e interdisciplinaria cualificó la propuesta preliminar fortaleciendo una mirada más amplia y contextualizada del modelo.

\section{Financiación}

Programa para la Disminución de la carga de la Enfermedad Crónica no Trasmisible en Colombia, con el apoyo financiero de COLCIENCIAS, código 501753730890.

\section{Referencias}

Achury M, Castaño H, Gómez L, Guevara N. Calidad de vida de los Cuidadores de pacientes con enfermedades crónicas con parcial dependencia. Investigación en Enfermería: Imagen y Desarrollo. 2011;13(1):27-46.

Agudelo C, Hoz F, Mojica M, Eslava J, Robledo R, Cifuentes P, et al. Prioridades de investigación en salud en Colombia: perspectiva de los investigadores. Revista Salud pública. 2009;11(2): 301-309. Recuperado de: http://www.scielosp.org/scielo. php?script=sci_arttext\&pid=S0124-00642009000200014\&lng

Alwan A, Etienne C. Prevention and management of chronic disease: a litmus test for health systems strengthening in low-income and middle-income countries. The Lancet. 2010;376(9754):7851797.

Ariza K, Isaza P, Gaviria AM, Quinceno JM, Vinaccia S, Alvarán L, et ál. Calidad de vida relacionada con la salud, factores psicológicos y fisiopatológicos en pacientes con diagnóstico de lupus eritematoso sistémico LES. Ter Psicol. 2010;28(1):27-36.

Baker D, Asch S, Keesey J, Brown J, Chan K, Joyce G, Keeler E. Differences in education, knowledge, self-management activities, and health outcomes for patients with heart failure cared for under the chronic disease model: the improving chronic illness care evaluation. J Card Fail. 2005;11(6):405-414.

Barrera L, Pinto N, Sánchez B. "Cuidando a los Cuidadores": Un programa De apoyo a familiares de personas con enfermedad crónica. Index de Enfermería. 2006;15(52-53):54-58.

Barrera L, Pinto N, Sánchez B. Habilidad de cuidado de cuidadores familiares de personas con enfermedad crónica: Comparación de géneros. Actualizaciones en Enfermería. 2006;9(2):9-13.
Barrera L, Pinto N, Sánchez B. Hacia la construcción de un modelo de cuidado de cuidadores de personas con enfermedad crónica. Actualizaciones en Enfermería. 2008; 11(2):23-29.

Barrera L, Pinto N, Sánchez B, Carrillo G, Chaparro L. (2010) Cuidando a los cuidadores familiares de personas con enfermedad crónica. Universidad Nacional de Colombia, Facultad de Enfermería.

Blum K, Sherman D. Understanding the experience of caregivers: a focus On transitions. Seminars in Oncology Nursing. 2010;26(4):243-258.

Borruel MA, Mas IP, Borruel GD. (2010). Estudio de carga de enfermedad: Argentina. Buenos Aires: Ministerio de Salud de la Nación.

Boykin A, Schoenhofer S. (2010). Nursing as caring: A model for transforming practice. Jones \& Bartlett Publishers.

Byock I, Norris K, Curtis R, Patrick D. Improving end-of-life experience and care in the community: a conceptual framework. J Pain Symptom Manage. 2001; 22(3):759-773.

Cameron J, Gignac M. Timing It Right: A conceptual framework for addressing the support needs of family caregivers to stroke survivors from the hospital to the home. Patient Educ Couns. 2008;70(3):305-314.

Cardenas D, Melenge B, Pinilla J, Carrillo GM, Chaparro L. Soporte social con el uso de las TIC para cuidadores de personas con enfermedad crónica: un estado del arte. Aquichan. 2010;10(3):204-213.

Carreño S. (2012). Metasíntesis de calidad de vida en cuidadores familiares de personas en situación de enfermedad crónica (Tesis de maestría). Universidad Nacional de Colombia, Bogotá.

Carrillo GM, Sánchez B, Barrera L. Habilidad de cuidado de cuidadores familiares de niños y adultos con enfermedad crónica. Index Enferm. 2014;23(3):129-133.

Carrillo GM, Chaparro L, Barrera L, Pinto N, Sánchez B. El blog como herramienta de soporte social para personas con enfermedad crónica. Ciencia y Enfermería. 2011; 17(3):137-149.

Cho EA. Proposed theoretical framework addressing the effects of informal Caregivers on health-related outcomes of elderly recipients in home health care. Nursing Research. 2007:1(1):2334.

Consejo Superior Universitario. (2011). Acuerdo 16 de 2011 del Consejo Superior Universitario. Por el cual se establece la Política Ambiental de la Universidad Nacional de Colombia. Universidad Nacional de Colombia.

Córdova VJ, Barriguete MJ, Lara EA, Barquera S, Rosas PM, Hernández AM, et al. Las enfermedades crónicas no transmisibles en México: sinopsis epidemiológica y prevención integral. Salud pública México. 2008;50(5):419-427.

Ferrell B, et al. Measurement of the quality of life in cancer survivors. Qual Life Res. 1995;4:523-531.

Gignac M, Cott C. A conceptual model of independence and dependence for adults with chronic physical illness and disability. Soc Sci Med . 1998;47(6):739-753. 
Gonzáles A, Fernández C, García G, Soler J, Arce C, Cueto J. Quality of life parameters in terminal oncology patients in a home car unit. Psychology in Spain. 2002; 6:68-76.

Grupo de Cuidado. 1998. Dimensiones del cuidado. Universidad Nacional de Colombia, Facultad de Enfermería. Grupo de Cuidado al paciente Crónico y su familia [Sede web]. (2006)www. gcronico.unal.edu.co. recuperado de:http://www.gcronico. unal.edu.co/programas.php

Hebert M, Korabek B, Scott R. Moving research into practice: A decision framework for integrating home telehealth into chronic illness care. Int J Med Inform. 2006;75(12):786-794.

Hobro N, Weinman J, Hankins M. Using the self-regulatory model to Cluster chronic pain patients: the first step towards identifying relevant treatments. Pain. 2004;108(3): 276-283.

Jova R, Rodríguez A, Díaz A, Balcindes S, Sosa I, De Vos P, Van der Stuyft P. Modelos de atención a pacientes con enfermedades crónicas no transmisibles en Cuba y el mundo. Medisan. 2011;15(11):1609-1620.

Kelvyn J, Craig D. Individuals and their ecologies: analyzing the geography Of chronic illness within a multilevel modeling framework. Health Place. 1995;1(1):27-40.

Lange I, Campos S, Urrutia M, Bustamante C, Alcayaga C, Tellez A, Piette J. Efecto de un modelo de apoyo telefónico en el auto-manejo y control metabólico de la Diabetes tipo 2, en un Centro de Atención Primaria, Santiago, Chile. Rev Méd Chile. 2010;138(6):729-737.

Lozada P, Aguinaga L, Páez R, Olmedo C, Pozo A. (1995). El peso de la enfermedad en Ecuador. Recuperado de: http://www. opecu.org/bevestre/revistas/CEPAR/pesoenf.pdf.

Lozano R, Franco F, Solís P. El peso de la enfermedad crónica de México. Salud Pública de México. 2007; 49(1):283-287.

Lucumí D, Gutiérrez A, Moreno J, Gómez L, Lagos N, Rosero M, et al. Planeación Local para enfrentar el Desafío de las Enfermedades Crónicas en Pasto, Colombia. Rev Salud pública. 2008;10(2):343-351.

Mayeroff M. 1990. On Caring. 1era Ed. United States: William Morrow Paperbacks.

Ministerio de salud de Chile, \& Pontificia Universidad Católica de Chile. Informe final Estudio de carga de enfermedad y carga atribuible, Chile 2007. Santiago de Chile: Informe del departamento de Salud Publica de la Universidad Católica de Chile; 2007.

Ministerio de salud del Perú. (2008). La carga de la Enfermedad y Lesiones en el Perú, Mortalidad, incidencias, prevalencias, duración de la enfermedad, discapacidad y años de vida saludables perdidos. Marzo 2008

Newman M. 2008. Transforming presence: the difference that nursing makes. Philadelphia:F.A. Davis.

Nkongho N. The caring ability inventory. Strickland OL and Waltz CF. Measurement of Nursing Outcomes: Self Care and Coping. New York: Springer Publishing Company. 1990;4:3-16.
Nolan M, Mock V. A conceptual framework for end-of-life care: a reconsideration of factors influencing the integrity of the human person. J Prof Nurs. 2004;20(6):351-360.

Organización Mundial de la Salud. 2012. Estadísticas Sanitarias Mundiales 2012. Informe de un Grupo Científico de la OMS. Serie de informes técnicos. Ginebra.

Organización Mundial de la Salud. 2014. Noncommunicable Diseases Country Profiles Informe técnico OMS: Ginebra.

Ministerio De Salud de la República de Colombia. 1993. Resolución No. 008430: Por la cual se establecen las normas científicas, técnicas y administrativas para la investigación en salud.

Rodríguez J, Acosta N, Peñaloza R. 2008. Carga de enfermedad Colombia 2005: resultados alcanzados. Documento Técnico Universidad Javeriana, Centro de Proyectos para el Desarrollo CENDEX: Bogotá, ASS/1502-08.

Roger G. The conceptual framework of palliative care applied to advanced Parkinson's Disease. Parkinsonism Rel Disord. 2012;18(3):2-5.

Rogers A. 2009. Consideraciones sobre la autogestión de las enfermedades crónicas. Indexde Enfermería, 18(4), 253-257. Recuperado de: http://scielo.isciii.es/scielo.php?pid=S1132$12962009000400009 \&$ script=sci_arttext

Samson A, Siam H. Adapting to major chronic illness: A proposal for a comprehensive task-model approach. Patient Education and Counseling. 2008;70(3):426-429.

Sánchez B. Cuidado seguro: la nueva tendencia en el cuidado de la salud. Actualizaciones en Enfermería. 2010;14(2):27-32.

Sánchez B, Carrillo G, Barrera L, Chaparro L. Carga del cuidado de la enfermedad crónica no transmisible. Aquichan. 2013;13(2):247-260.

Struk C, Moss J. Focus on Technology: What Can you Do to Move the Vision Forward?.Comput In form Nurs. 2009;27(3):193.

Vinaccia S, Orozco M. Aspectos psicosociales asociados con la calidad de vida de personas con enfermedades crónicas. Diversitas. 2005:1(2):125-137.

Walker J, Jackson H, Littlejohn G. Models of adjustment to chronic illness: Using the example of rheumatoid arthritis. Clin Psychol Rev. 2004;24(4):461-488.

Wagner J, Heapy A, Frantsve L, Abbott G, Burg M. Transtheoretical Model constructs in smokers with and without medical illness: A second look at the medical effect. Addict Behavioral. 2006;31(7):1283-1289.

Washington K, Meadows S, Elliott S, Koopman R. Patient Information needs of informal caregivers of older adults with chronic health conditions. Education and Counseling. 2011;83(1):3744.

World Health Organization. 2014. Measuring Quality of Life. Recuperado de: http://www.who.int/mental_healt/../68.pdf 